\title{
Le rythme et la sueur « À bordeaux, il n'y a plus d'âme. il faut en recréer une »
}

\section{Olivier Camus}

\section{(2) OpenEdition}

1 Journals

Édition électronique

URL : http://journals.openedition.org/communicationorganisation/2007

DOI : 10.4000/communicationorganisation.2007

ISSN : $1775-3546$

Éditeur

Presses universitaires de Bordeaux

\section{Édition imprimée}

Date de publication : 1 novembre 1997

ISSN : 1168-5549

\section{Référence électronique}

Olivier Camus, « Le rythme et la sueur « À bordeaux, il n'y a plus d'âme. il faut en recréer une » », Communication et organisation [En ligne], 12 | 1997, mis en ligne le 26 mars 2012, consulté le 26 avril 2019. URL : http://journals.openedition.org/communicationorganisation/2007 ; DOI : 10.4000/ communicationorganisation.2007

Ce document a été généré automatiquement le 26 avril 2019

(c) Presses universitaires de Bordeaux 


\title{
Le rythme et la sueur « À bordeaux, il n'y a plus d'âme. il faut en recréer une »
}

\author{
Olivier Camus
}

1 «À Bordeaux, il n'y a plus d'âme. Il faut en recréer une ». Vaste programme... Notre propos sera donc de montrer ici les conditions nécessaires à la création d'une âme.

2 Nous dansons et nous transpirons en rythme. Nous travaillons et nous transpirons toujours en rythme. Aux deux pôles de l'activité humaine, le travail et la fête, nous ne pouvons séparer le rythme et la sueur, nous ne pouvons envisager l'une ou l'autre de ces activités sans ces deux éléments. Le rythme et la sueur. Tous deux sont créateurs de lien social. Tous deux sont les ingrédients indispensables de notre délicate et précieuse recette.

3 Donc... « à Bordeaux, il n'y a plus d'âme. Il faut en recréer une »¹.

4 C'est Gernot Rhor qui dresse le constat et s'attelle à la tâche. Le contexte ? L'équipe de football des Girondins de Bordeaux traverse une période de marasme dans le championnat de France 1995/1996, bien que toujours qualifiée en coupe d'Europe de l'U.E.F.A. Le nouvel entraîneur Gernot Rhor, reprenant en cours de saison l'équipe dirigée jusqu'alors par Slavo Muslin, n'a rétrospectivement pas failli, il a su insuffler une âme à son équipe, lui permettant d'aller brillamment jusqu'en finale de cette coupe d'Europe, après avoir éliminé le grand Milan A.C.

En l'occurence, c'est d'âme collective dont il s'agit.

6 Vous allez me dire que le langage courant galvaude la notion d'âme et possède une grande capacité d'exagération. Il s'agit pourtant bel et bien d'une manifestation de l'âme collective. Dans sa perspective collective, cette âme-là a besoin de sentiments et buts communs, éléments indispensables, pour parvenir à un état d'effervescence, état qui permettra à l'âme collective d'apparaître. Dans le même temps, sans cette âme collective, il n'y aurait point d'effervescence. 
7 L'effervescence, comme l'a suffisamment bien montré Emile Durkheim², est un état de conscience collectif qui gouverne aussi bien la cérémonie religieuse que la fête profane, l'émeute populaire ou certaines manifestations de foules etc. L'effervescence est une façon, selon l'auteur, de permettre à la société de devenir une «machine à faire des dieux » et de déguiser de cette façon le « divin social » qu'elle porte. L'effervescence est donc un état qui nous " soulève au-dessus de notre niveau ordinaire » et dont la chaleur et la passion commune sont les indispensables « matières premières ".

8 Notre propos est d'évoquer ces ingrédients indispensables à l'effervescence pour que celle-ci permette aux participants de sentir leur âme collective.

Revenons-en maintenant à notre exemple de circonstances. Quelques mois avant d'être occupés à se recréer une âme, les Girondins de Bordeaux avait commencé tôt leur saison. Pour avoir raté la qualification à une coupe européenne (la coupe de l'U.E.EA.) de peu, ils essaient de la rejoindre par le biais d'une épreuve préliminaire, la Coupe Intertoto rassemblant des clubs européens ayant raté l'accessit direct à cette compétition plus prestigieuse.

Ce soir-là, début juillet, au Parc Lescure, l'équipe des Girondins «se promène ». L'IFK Norrköping (Suède), l'adversaire du jour, prend l'eau de toutes parts et, plus d'une fois déjà, leur gardien de but a dû se contenter d'aller pêcher les ballons au fond de ses filets. Le score monte jusqu'à un débridé 6-2, dont un doublé de Zinedine Zidane (7e et $64 \mathrm{e}$ minutes) et un triplé de l'avant-centre Tholot (5e, $15 \mathrm{e}$ et $56 \mathrm{e}$ minutes).

11 Devant le poste de télévision, grâce aux micros d'ambiance disposés autour du terrain, on entend le public (pourtant clairsemé) qui encourage son équipe, on entend les bruits que font les pieds quand ils frappent la balle, on entend les cris des joueurs derrière le commentaire tel qu'en lui-même des consultants de TF1. Derrière, on entend aussi Slavo Muslin, l'entraîneur bordelais, donner de la voix pour dire à ses joueurs encore et toujours le sens du jeu. On ne voit même pas Muslin à l'écran, on l'entend, on le devine debout près de son banc. Et que dit-il ? On l'entend en permanence crier « fais tourner !", «Fais tourner! », «Fais tourner!»

12 Il parle du ballon bien sûr. Nous noterons donc que le ballon est un de ces objets qui «tournent ». Mais ici, en l'occurence, il n'est pas question de sa rondeur ni du fait qu'il roule. Comme un rythme, il tourne. Comme ce joint de cannabis qui fait tant jaser le milieu sportif depuis quelques mois, il passe de l'un à l'autre. Le ballon «tourne » d'un joueur à l'autre. Le ballon tisse le lien entre les joueurs de l'équipe et donne au jeu sa dimension collective. La circulation du ballon assure le passage permanent de l'individuel au collectif.

13 Le football est un autre exemple de ces activités où l'homme verse sa sueur en rythme. La sueur? Eh bien, les joueurs doivent «mouiller le maillot». Le rythme? La vigilance de l'adversaire ne sera prise à défaut que si la circulation du ballon et les actions individuelles possèdent suffisamment de rythme. C'est-à-dire : vitesse et «timing ». À trop trainer sur le chemin de l'offensive, la défense peut se replacer. À trop se précipiter, on perd le contrôle du ballon. Le «timing » : la balle est en mouvement, le pied ou la tête doit la frapper au millième de seconde, un temps de retard et la balle « dévisse » et va se perdre dans les airs au lieu de fendre l'air vers le but.

14 Avant d'être musical, le rythme est déjà la simple coordination des gestes. Avant que n'existent l'industrie et les chaînes de montage, déjà l'homme au travail aligne sa cadence sur celle de ses «collègues $»^{3}$. Ensemble, ils synchronisent leurs gestes pour ne pas se 
gêner, pour ne pas empiéter sur ceux du voisin. Le rythme devient la condition de l'efficacité de leur besogne, il introduit un ordre ${ }^{4}$.

L'activité sociale par ce qu'elle a de «but commun » repose toujours sur une construction rythmique. La communication s'engage et se poursuit en reposant sur un rythme, succession de creux et de plein, de parole et de silence, sur le passage d'un interlocuteur à l'autre.

16 Le rythme est créateur de l'espace et du temps 5 , il est une "construction sociale de la réalité ». Le rythme est aussi le «but commun » et permet, à la fois, d'être ensemble et à sa place. Le musicien Ray Lema nous en donne un exemple en présentant un village réunit autour de l'exécution d'un rythme sur des tambours, c'est la « Roue zaïroise » dont il nous donne la philosophie : «tu prends une bouteille sur laquelle tu frappes, dans un groupe, personne ne va te demander dans quelle gamme tu joues. Ca n'a aucune importance quand la pulsation est là. Si tu es dans le temps, c'est tout bon! Ainsi chacun doit respecter le temps, s'intégrer dans le cercle musical qui tourne, sans toucher les autres. $\mathrm{Tu}$ dois trouver ta place dans les vides laissés par les autres musiciens. $»^{6}$

17 La communication avec l'autre se fait dans un cadre rythmique au travail comme à la fête. Leroi-Gourhan en a relevé l'importance, "dans le dépaysement mental, il faut reconnaître que les ruptures de l'équilibre rythmique jouent un rôle important $»^{7}$. Ainsi pour entrer dans le cercle esthético-religieux, nous sortons du «cercle normal » sociotechnique.

18 Marcel Mauss, lui, a su relever ces caractères dans la fête religieuse. « Tout le corps social est animé d'un même mouvement. (...) Ce mouvement rythmique est l'expression immédiate d'un état mental où la conscience de chacun est accaparée par un seul sentiment, une seule idée, hallucinante, celle du but commun $»^{8}$. Alors qu'isolé, il « traîne languissamment sa vie quotidienne $"^{9}$, c'est ce moment où l'individu bascule à la fois dans un autre champ de la vie sociale et dans un autre état de conscience qui nous intéresse ici. Ce moment est celui de l'effervescence, moment où apparaît l'âme collective.

19 L'effervescence est ambivalente, festive ou guerrière, et se manifeste de mille et une façons dans la vie sociale, nous avons ici retenu les exemples du sport et de la fête musicale. Dans un cas comme dans l'autre disions-nous, le rythme et la sueur sont le chemin qui conduit vers l'âme...

20 Une recherche sur la musique funk afro-américaine ${ }^{10}$ nous a révélé que l'étymologie du mot africain signifiait "transpiration positive", avec, sous-entendue, l'idée de participation collective à l'événement. En anglais, «funk» a d'abord désigné la sueur froide, celle de l'esclave noir face à son maître, ainsi que les diverses sécrétions du corps que l'hygiène s'emploie à éliminer. Puis, comme souvent dans les cultures populaires, par un processus de double négation ${ }^{11}$, le terme péjoratif est devenu positif. Par le Funk, on triomphe de la peur qui auparavant nous tenaillait et le corps s'y dépense, chaud et pour la humide. Comme le vin des rites dionysiaques, c'est la conjonction du chaud et de l'humide qui unit les participants dans un même élan rythmique. Le Funk, dansant par vocation, mieux qu'aucune autre musique de ce siècle nous montre l'importance et le rôle de la sueur dans la fête.

21 C'est à partir de ces observations que nous avons proposé la notion d'âme-sueur pour évoquer cette âme collective qui se manifeste dans l'effervescence ${ }^{12}$. 

collective, c'est bel et bien d'âme-sueur qu'il s'agit. Il n'y a pas grand-chose à ajouter, ni guère de conseils à donner : nul doute que l'entraîneur actuel connaît la recette.

\section{NOTES}

1. Entretien Gernot Rhor, France Football, 6-2-1996

2. Emile Durkheim, Les Formes élémentaires de la vie religieuse, Paris, Le livre de poche. Librairie Générale Française, 1991 pour la présente édition

3. Evidemment on imagine plutôt qu'il est ici question d'un travail physique, celui de l'ouvrier, de l'artisan, du paysan etc.

4. Roger Bastide, Art et société, Paris, Payot, 1977, p. 53 et 71 par exemple où il fait référence aux travaux de K. Bücher, R. Wallaschek et F. B Gummère.

5. André Leroi-Gourhan, Le Geste et la parole Tome 2 : La Mémoire et les rythmes. Paris, Albin Michel, Coll. Sciences d'aujourd'hui, 1965, p. 136

6. Entretien Ray Lema in Bruno Tuliefte et Njami Simon, Ethnicolor (Musique, mode, littérature, pub. cinéma: influences réciproques Afrique I France), Paris, Ed Autrement, 1987 Cf. aussi le film de Philippe Conrath, Paris, c'est l'Afrique, où Ray Lema y développe également la « philososphie de la roue zaïroise ».

7. André Leroi-Gourhan, ibid, pp. 99-100

8. Marcel Mauss, «Esquisse d'une théorie générale de la magie (Les états collectifs et les forces collectives) », Sociologie et anthropologie, Paris, PUF, Quadrige, 1950, p. 126

9. Emile Durkheim, ibid., p. 385

10. Olivier Cathus. Quelques aspects des musiques populaires en général et de du Funk en particulier, Thèse de doctorat en sociologie sous la direction de Michel Maffesoli, 1997

11. Sur le sens de la 'double-négation se référer a Gilbert Durand, Les Strucutres anthropologiques de l'imaginaire. Paris, Dunod, 1992 pour la présente $\left(11^{\mathrm{e}}\right)$ édition.

12. Olivier Cathus, L'âme-sueur (Funk et effervescence) ». Sociétés n'46, Paris, Dunod, 1994

\section{AUTEUR}

\section{OLIVIER CAMUS}

Université Paris V. Centre d'études sur l'actuel et le quotidien 